\title{
Proof of Concept of a Simple Computer-Assisted Technique for Correcting Bone Deformities
}

\author{
Burton $\mathrm{Ma}^{1}$, Amber L. Simpson ${ }^{2}$, and Randy E. Ellis ${ }^{1,2}$ \\ ${ }^{1}$ Human Mobility Research Centre, Kingston General Hospital, Kingston, Ontario, Canada \\ ${ }^{2}$ Queen's University, Kingston, Ontario, Canada \\ mab, simpson, ellis@cs.queensu.ca
}

\begin{abstract}
We propose a computer-assisted technique for correcting bone deformities using the Ilizarov method. Our technique is an improvement over prior art in that it does not require a tracking system, navigation hardware and software, or intraoperative registration. Instead, we rely on a postoperative CT scan to obtain all of the information necessary to plan the correction and compute a correction schedule for the patient. Our laboratory experiments using plastic phantoms produced deformity corrections accurate to within $3.0^{\circ}$ of rotation and $1 \mathrm{~mm}$ of lengthening.
\end{abstract}

\section{Introduction}

Ilizarov's method, an orthopaedic surgery used to correct deformities of the long bones, uses external fixation apparatus to apply controlled stress to a cut or fractured bone; the body's response is to regenerate the bone and soft tissues and grow in the direction of the applied stress. By maintaining and controlling the direction of the tensile load over a period of time a wide range of deformities can be corrected [1]. We propose a computer-assisted approach that can be used at any institute having modest computing capabilities, as it does not require preoperative $\mathrm{CT}$, intraoperative registration, or intraoperative navigation.

The Taylor Spatial Frame (Smith \& Nephew, Memphis, TN) is one type of external fixator used for Ilizarov's method. It is made up of two rings connected by six telescoping struts; changing the strut lengths causes relative motion between the rings. The rings are fixed to the patient using thin, tensioned Kirschner wires or wider Steinman pins drilled into the bone through the skin and surrounding soft tissues.

Conventional planning of the correction is performed using radiographs and measurements of deformity assessed in clinic. Thirteen parameters must be measured/set by the surgeon when using the Taylor Spatial Frame.

The goal when using the Taylor spatial frame is to mount the frame on the patient so that it mimics the shape of the deformity (ie. the distal ring should be fixed parallel to the plane of the most distal joint and similarly for the proximal ring and joint). If this is done properly, the frame will be in a neutral configuration (all struts equal in length) when the deformity is corrected. If the frame is not mounted properly then there will be a residual deformity if the frame is restored to its neutral configuration.

Two computer-assisted techniques using three-dimensional planning have previously been described. Iyun and colleagues [2] described a system that used surface models 
computed from CT to plan the correction and the placement of the Steinman pins for mounting the rings. They proposed to use intraoperative registration and navigation to implant the pins. The primary drawback of this approach is that the preoperative choice of pin placement may be incompatible with intraoperative conditions.

Simpson and coauthors [3] described a CT-based technique that required intraoperative registration to establish the relationship of the rings with respect to the bone. Using this information in conjunction with a preoperative plan allowed them to compute a correction schedule for the patient. Their approach eliminated the two major sources of error in the conventional technique (planning on radiographs and intraoperative mounting of the rings to mimic the deformity) but required an intraoperative tracking system and segmentation of the CT scan to produce the models for planning and registration. Their technique has been used clinically [4].

We use a postoperative CT scan of the bone and frame to (1) establish the relationship between the bone and the rings, and (2) plan the required correction. We avoid segmenting the CT scan by using direct volume visualization for planning purposes. Kirschner wires should be preferred over Steinman pins when using our proposed technique; the relatively wide stainless steel Steinman pins tend to produce excessive noise in the CT scan. The pins do not interfere with planning if they are located far from the joint lines.

\section{Method}

Our proposed technique would be applied in seven steps:

1. Preoperative patient care would proceed as with conventional technique. The surgeon would not need to plan the procedure on radiographs, but may do so if desired.

2. Intraoperative patient care would proceed as with conventional technique. If the surgeon chooses to follow a conventional preoperative plan then the rings should be mounted to mimic the deformity. If the surgeon chooses to rely on the computerassisted technique then the rings could be mounted in any reasonable configuration.

3. Postoperative patient care would be modified to include a CT scan of the limb and frame. The scan can occur at any time during the correction phase if preoperative planning has been performed and a correction schedule obtained; it must occur before the correction phase begins if using only the computer-assisted technique because we compute the correction schedule as the last step of our method. The usual 10-day period of callus formation (before manipulation of the frame begins) provides ample time to perform the scan.

4. Sets of at least 3 landmarks $\left\{F_{p}\right\}$ and $\left\{F_{d}\right\}$ on the proximal and distal rings are located in the CT scan; our tests used a central point on the tabs of the rings (see Figure 1 but other landmarks such as the strut attachment points or fiduciary markers machined into or attached onto the rings could be used. Models of the rings would be registered to the CT landmarks $\left\{F_{p}\right\}$ and $\left\{F_{d}\right\}$ to yield the rigid transformations $\mathbf{T}_{p}$ and $\mathbf{T}_{d}$ (see Section 2.1.

5. A reference point $\mathbf{x}_{\mathrm{ref}}$ is identified in the CT scan; typically, $\mathbf{x}_{\mathrm{ref}}$ is chosen as the point in the middle of the bone on the plane of the osteotomy. The reference point is used to control the rate of the correction schedule. 


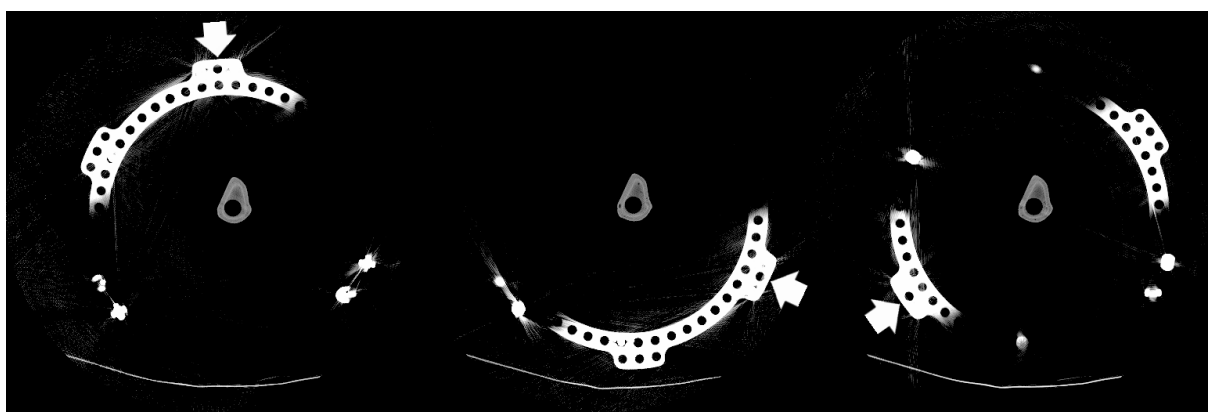

Fig. 1. Proximal ring landmarks. We use the central point on the outer diameter of the three tabs where the struts attach to the ring. The distal ring landmarks are similar.

6. Direct volume rendering of the CT scan can be used for planning purposes. Only simple operations, such as selecting a region of interest and cutting of the volume into proximal and distal fragments, are required. The proximal fragment is moved to plan the correction because orthopaedic convention views the distal end as stationary; however, our technique can easily be modified to allow movement of both fragments. The rigid transformation $\mathbf{T}_{\text {plan }}$ of the proximal fragment in CT coordinates is recorded (see Section 2.2).

7. Using the reference point $\mathbf{x}_{\text {ref }}$ and the transformations $\mathbf{T}_{p}, \mathbf{T}_{d}$, and $\mathbf{T}_{\mathrm{plan}}$ a correction schedule is calculated as described in Section 2.3. Note that $\mathbf{x}_{\text {ref }}$ and $\mathbf{T}_{\text {plan }}$ are defined in CT coordinates, and $\mathbf{T}_{p}$ and $\mathbf{T}_{d}$ map model points into CT coordinates; thus, the calculation of the correction schedule takes place in a single (CT) coordinate system.

\subsection{Ring Registration}

Our models of the proximal and distal rings and the centers of rotation of the universal joints of the struts (the strut end points) relative to the rings are shown in Figure 2 and numerical details are given in Table 1 Registration of the model proximal and distal landmarks $\left\{M_{p}\right\}$ and $\left\{M_{d}\right\}$ to the fiduciary CT landmarks $\left\{F_{p}\right\}$ and $\left\{F_{d}\right\}$ can be estimated by any absolute orientation solver; we use Horn's method [5]. Using the registration transformations $\mathbf{T}_{p}$ and $\mathbf{T}_{d}$ we can compute the current strut lengths as

$$
s_{i}=\left(\left(\mathbf{T}_{p} \mathbf{p}_{i}-\mathbf{T}_{d} \mathbf{d}_{i}\right) \cdot\left(\mathbf{T}_{p} \mathbf{p}_{i}-\mathbf{T}_{d} \mathbf{d}_{i}\right)\right)^{1 / 2}, i=1 . .6
$$

where $\mathbf{T}_{p} \mathbf{p}_{i}$ and $\mathbf{T}_{d} \mathbf{d}_{i}$ ) are the proximal and distal model strut end points, respectively, registered to CT coordinates. The estimated current strut lengths can be compared against the physical strut lengths to validate the registration process.

\subsection{Planning}

We use direct volume visualization of the CT scan to avoid segmentation. The rings, wires, and pins can, for the most part, be removed from the images by using simple region of interest selection. The scan can be separated easily into two volumes of slices 


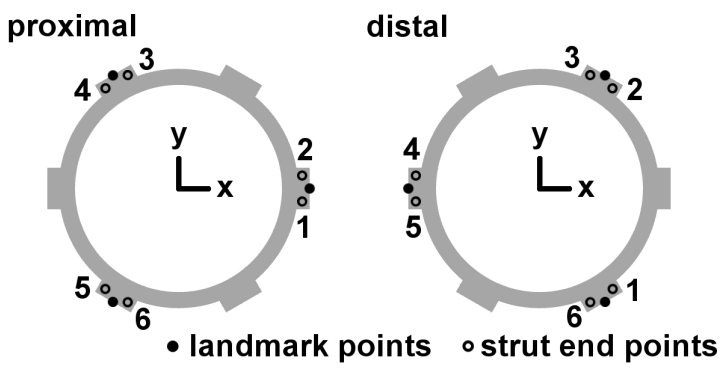

Fig. 2. Models of the $180 \mathrm{~mm}$ proximal and distal rings and the strut end points

Table 1. Cylindrical coordinates (radius, angle, $z$ ) of ring landmarks and strut end points for the $180 \mathrm{~mm}$ ring

\begin{tabular}{|c|c|c|}
\hline & Proximal Ring & Distal Ring \\
\hline Landmarks & $\left(116.4 \mathrm{~mm}, 0^{\circ}, 0 \mathrm{~mm}\right)$ & $\left(116.4 \mathrm{~mm}, 60^{\circ}, 0 \mathrm{~mm}\right)$ \\
& $\left(116.4,120^{\circ}, 0\right)$ & $\left(116.4,180^{\circ}, 0\right)$ \\
& $\left(116.4,240^{\circ}, 0\right)$ & $\left(116.4,300^{\circ}, 0\right)$ \\
\hline Strut End Points & $\left(109.5 \mathrm{~mm}, 0-6.67^{\circ},-16 \mathrm{~mm}\right)$ & $\left(109.5 \mathrm{~mm}, 300+6.67^{\circ}, 16 \mathrm{~mm}\right)$ \\
& $\left(109.5,0+6.67^{\circ},-16\right)$ & $\left(109.5,60-6.67^{\circ}, 16\right)$ \\
& $\left(109.5,120-6.67^{\circ},-16\right)$ & $\left(109.5,60+6.67^{\circ}, 16\right)$ \\
& $\left(109.5,120+6.67^{\circ},-16\right)$ & $\left(109.5,180-6.67^{\circ}, 16\right)$ \\
& $\left(109.5,240-6.67^{\circ},-16\right)$ & $\left(109.5,180+6.67^{\circ}, 16\right)$ \\
& $\left(109.5,240+6.67^{\circ},-16\right)$ & $\left(109.5,300-6.67^{\circ}, 16\right)$ \\
\hline
\end{tabular}

that are, respectively, proximal and distal to the osteotomy plane. The proximal volume is manipulated to achieve the desired correction.

Our planning software, written using VTK (wWw . vtk . org), provides three orthogonal views of the two volumes (Figure 3). On a PC with modest computing power, it maintains interactive rates with the volumes we used in this study. The output of the planning software is the rigid transformation $\mathbf{T}_{\text {plan }}$, in CT coordinates, of the proximal fragment.

\subsection{Correction Schedule}

The correction schedule specifies the daily strut lengths needed to achieve the desired correction. The patient adjusts each strut to the specified length once each day.

The current (time $t=0$ ) and final (time $t=n$ ) locations of the model proximal strut end points in CT coordinates are given by

$$
\begin{aligned}
\mathbf{p}_{i, t=0}^{C T} & =\mathbf{T}_{p} \mathbf{p}_{i} \\
\mathbf{p}_{i, t=n}^{C T} & =\mathbf{T}_{\text {plan }} \mathbf{T}_{p} \mathbf{p}_{i} .
\end{aligned}
$$

${ }^{1}$ CPU: AMD 3800 X2, GPU: NVIDIA 7800GS. 


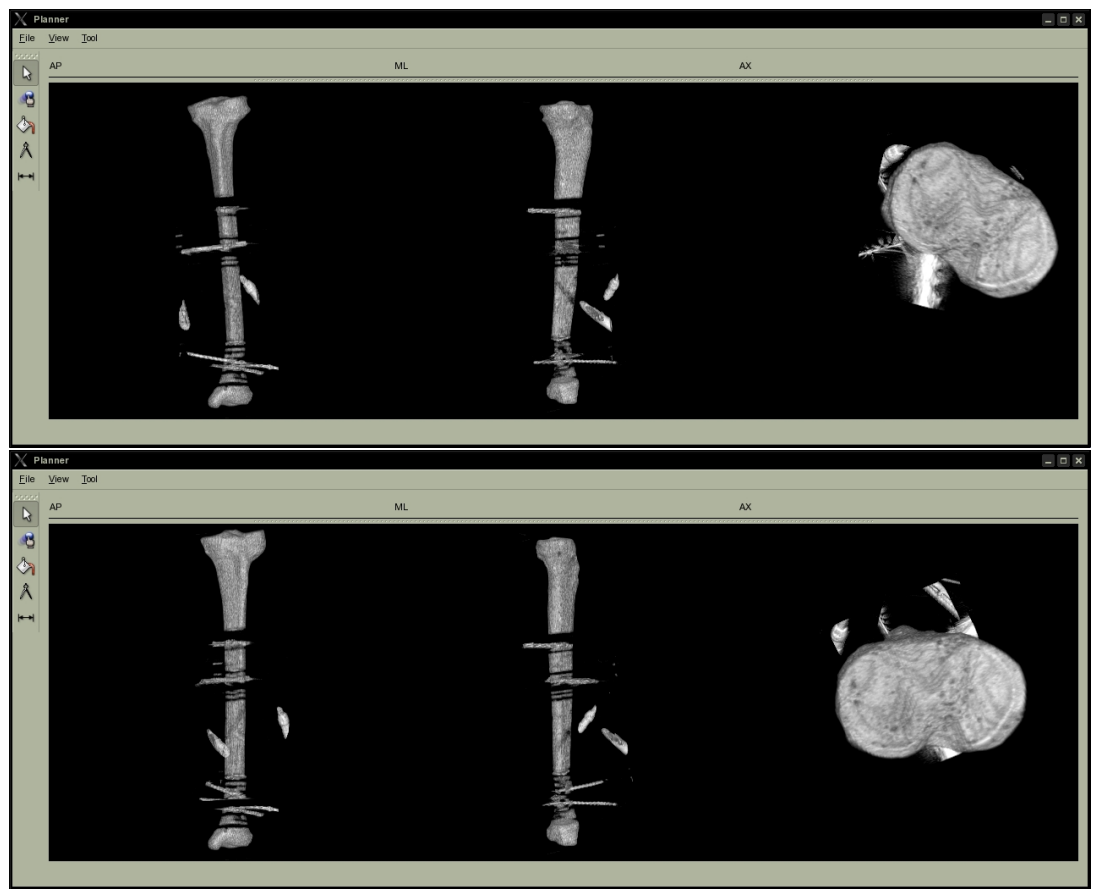

Fig. 3. Volume rendered planning images for phantom 3 before (top) and after (bottom) deformity correction with the distal end held stationary; note the large axial rotation deformity. Images were generated using a linear opacity transfer function and a constant color transfer function; no attempts were made to optimize the transfer functions.

Normally, the duration $n$, measured in days, is not given. Instead, the reference point $\mathbf{x}_{\text {ref }}$ is displaced by a specified distance each day; typically, the magnitude of the displacement is $1 \mathrm{~mm}$ per day.

We compute the correction schedule by converting $\mathbf{T}_{\text {plan }}$ to its screw representation [6]. A screw transformation is a rotation of angle $\Theta$ about an axis with direction b passing through the point $\mathbf{c}$, followed by translation of magnitude $M$ along the same axis. We use brute force, discrete search over $\Theta$ and $M$ to find the strut lengths:

- find the screw parameterized by $\mathbf{b}, \mathbf{c}, \Theta$ and $M$ corresponding to $\mathbf{T}_{\text {plan }}$

- set $d \theta=\Theta / N, d M=M / N$ for some large $N$ (say $N=1000$ )

- set $\mathbf{x}_{\text {old }}=\mathbf{x}_{\text {ref }}$

- set $t=1$

- for $i=1 . . N$

- set $\theta_{i}=i \times d \theta$

- set $m_{i}=i \times d M$

- $\operatorname{set} \mathbf{T}_{i}=S\left(\mathbf{b}, \mathbf{c}, \theta_{i}, m_{i}\right)$

- set $\mathbf{x}_{i}=\mathbf{T}_{i} \mathbf{x}_{\text {ref }}$

- if $\left\|\mathbf{x}_{i}-\mathbf{x}_{\text {old }}\right\| \geq 1 \mathrm{~mm}$

* compute strut lengths for day $t$ (Equation 1 substitute $\mathbf{T}_{i} \mathbf{T}_{p}$ for $\mathbf{T}_{p}$ ) 
- end if

$*$ set $t=t+1$

$*$ set $\mathbf{x}_{\text {old }}=\mathbf{x}_{i}$

- end for

- compute strut lengths for the last day $t$ (Equation 1 substitute $\mathbf{T}_{\text {plan }} \mathbf{T}_{p}$ for $\mathbf{T}_{p}$ )

where $S(\mathbf{b}, \mathbf{c}, \theta, m)$ is the $4 \times 4$ rigid transformation matrix corresponding to the screw transformation with angle $\theta$, translation magnitude $m$, and axis with direction $\mathbf{b}$ passing through the point $\mathbf{c}$.

\subsection{Experimental Validation}

Three plastic tibia phantoms were obtained (Sawbones, Pacific Research Laboratories, Inc., Vashon, WA, USA). Two phantoms were of deformed bones with approximately $10^{\circ}$ varus proximal deformity at the level of the fibular head. The third bone was a normal tibia modified to have an approximately $45^{\circ}$ axial deformity at the distal end.

Each phantom was scanned using CT for validation purposes and polygonal surface models were created. A Taylor frame with $180 \mathrm{~mm}$ rings was mounted on each phantom using Kirschner wires on the ring closest to a joint line and Steinman pins on the other ring. The frame and phantom were scanned using CT with a slice spacing of $2.5 \mathrm{~mm}$. Our seven-step technique described at the beginning of the Methods section was used on phantoms 1 and 2 (varus proximal deformity) a total of six times, and on phantom 3 (axial distal deformity) four times. We compared the physical and computed strut lengths to validate the ring registration process (see Section 2.1).

The CT scan of the first phantom was performed with the phantom cut and distracted part-way through a correction schedule, which simulated a partial correction using conventional technique. The other phantoms were scanned before they were cut.

The accuracy of each correction was computed by registering the distal and proximal fragments to the surface model of the intact phantom. An Optotrak optical tracking system (Northern Digital Inc., Waterloo, Ontario, Canada) was used to acquire registration data. A dynamic reference body was attached to the distal fragment of the phantom. Registration points were collected from the surface of the phantom using a calibrated stylus. Let $\mathbf{T}_{\text {reg }, p}$ and $\mathbf{T}_{\text {reg, }, d}$ be the registration transformations from the tracker coordinate system to the CT coordinate system of the proximal and distal fragments, respectively. Then we have

$$
\mathbf{T}_{\text {achieved }}=\mathbf{T}_{\text {reg }, d} \mathbf{T}_{\text {reg }, p}^{-1} \approx \mathbf{T}_{\text {plan }}
$$

for the proximal fragment. The error between the achieved correction and the plan is

$$
\Delta=\mathbf{T}_{\text {plan }} \mathbf{T}_{\text {achieved }}^{-1}
$$

The total angular error of the achieved correction can be computed by finding the quaternion or screw representation of $\Delta$. The translational error can be computed by locating a relevant anatomic landmark $\mathbf{t}$ in the $\mathrm{CT}$ images and calculating

$$
\delta=\mathbf{T}_{\text {plan }} \mathbf{t}-\mathbf{T}_{\text {achieved }} \mathbf{t}
$$

We set $\mathbf{t}$ to be the location of the tibial intercondylar eminence in the CT images. 
Table 2. Errors of the achieved corrections

\begin{tabular}{|l|c|c|c|}
\hline & Femur 1 & Femur 2 & Femur 3 \\
\hline Total rotation error & $1.7^{\circ}, 3.0^{\circ}, 2.8^{\circ}, 2.4^{\circ}$ & $3.0^{\circ}, 2.2^{\circ}$ & $3.3^{\circ}, 3.0^{\circ}, 3.0^{\circ}, 3.2^{\circ}$ \\
\hline Lengthening error $(\mathrm{mm})$ & $-0.6,2.6,0.6,1.5$ & $1.0,1.0$ & $0.3,0.5,1.2,1.4$ \\
\hline
\end{tabular}

\section{Results}

The residual rotation and translation errors of the achieved correction are summarized in Table 2 The average rotational error was $2.8^{\circ}$ with no error greater than $3.3^{\circ}$. The average lengthening error was $1.0 \mathrm{~mm}$ with no error greater than $2.6 \mathrm{~mm}$. Our correction error results are comparable to clinical results reported by Feldman [7].

We did not experience any stress loading of the phantoms that would have caused unusual translational errors as reported in [3]. We did not observe differences between the computed and physical strut lengths of greater than $3 \mathrm{~mm}$ in any of the trials.

\section{Discussion}

Our technique aims to remove the most significant sources of error experienced with conventional technique. Our method does not require preoperative measurements on radiographs and it allows the surgeon to fix the rings on the patient in any reasonable configuration. Achieving these goals requires the use of a postoperative CT scan, which is conventionally not required.

The CT-based methods described by [2] and [3] also address the errors associated with conventional technique. The residual errors we measured were comparable to those reported by both [2] and [3]. Like their methods, ours yields the current strut lengths that can be compared to the physical strut lengths to ensure that the necessary measurements have been performed accurately. Unlike the other methods, ours does not require a tracking system, navigation hardware and software, intraoperative registration, or segmentation of the CT scan. Our method also has the advantage of being usable anytime before the correction schedule has been completed; thus our method can be used as the primary means of achieving a correction, or as a method to possibly recover from a failing conventional procedure. Because finding the ring landmarks and planning the correction can be accomplished quickly, it is possible to use our method for trauma cases if CT is available within approximately ten days of frame attachment.

The major disadvantage of our method is that the fidelity of the postoperative CT scan is compromised by the stainless steel hardware of the pins, wires, and small components of the frame. The imaging artifacts do not adversely affect the localization of the frame landmarks, but they can be an impediment to precise planning of the correction. The imaging artifacts are especially problematic if one of the bone fragments is small because it becomes difficult to visualize the correction in the degraded images; Steinman pins must be avoided in these circumstances. It is possible to use radiographs and clinical evaluation of axial rotation to assist in the planning process, but we have not yet evaluated the efficacy of such an approach. 
We used Horn's method to register the models of the rings to the ring landmarks localized in the CT images. A method such as the one described by Ohta and Kanatani [8] may prove to be more accurate because the CT voxel spacing is often anisotropic. The accuracy of our technique is dependent on the localization error of the ring landmarks, which is affected by the CT slice spacing. The errors introduced by the CT resolution could be minimized by designing special fiducial markers or registering the image of the rings (instead of identifying point landmarks).

A weakness of our current study is that it was performed using polyurethane phantoms that were very radiolucent. Imaging of biological specimens needs to be performed to determine if the fidelity of the CT scan is sufficient for reliable planning. Allowing for these limitations, our navigation-free technique provides clinicians with a novel application of computer-assisted orthopaedic surgery. Potential future work might include estimating the relationship of the rings with respect to the bone by using radiographs instead of the postoperative CT scan.

\section{References}

1. Lieverman, J.R., Friedlaender, G.E. (eds.): The Ilizarov technique for bone regeneration and repair. In: Bone regeneration and repair: biology and clinical applications, Humana Press, Totowa (2005)

2. Iyun, O., Borschneck, D.P., Ellis, R.E.: Computer-assisted correction of bone deformities using a 6-DOF parallel spatial mechanism. In: Niessen, W.J., Viergever, M.A. (eds.) MICCAI 2001. LNCS, vol. 2208, pp. 232-240. Springer, Heidelberg (2001)

3. Simpson, A.L., Ma, B., Borshneck, D.P., Ellis, R.E.: Computer-assisted deformity correction using the Ilizarov method. In: Duncan, J.S., Gerig, G. (eds.) MICCAI 2005. LNCS, vol. 3749 , pp. 459-466. Springer, Heidelberg (2005)

4. Simpson, A.L., Ma, B., Slagel, B., Borshneck, D.P., Ellis, R.E.: Computer-assisted distraction osteogenesis using the Taylor frame: initial clinical experiences. In: The 2nd Annual Congress of The British Society For Computer Aided Orthopaedic Surgery (2007)

5. Horn, B.K.P.: Closed-form solution of absolute orientation using unit quaternions. Journal of the Optical Society of America A 4, 629-642 (1987)

6. McCarthy, J.M.: An Introduction to Theoretical Kinematics. MIT Press, Cambridge (1990)

7. Feldman, D.S., Shin, S.S., Madan, S., Koval, K.: Correction of tibial malunion and nonunion with six-axis analysis deformity correction using the taylor spatial frame. Journal of Orthopaedic Trauma 17(8), 549-554 (2003)

8. Ohta, N., Kanatani, K.: Optimal estimation of three-dimensional rotation and reliability evaluation. IEICE Transactions on Information and Systems E82-D(11), 1247-1252 (1998) 